\title{
Généricité Dans Lullaby De J. M. G. Le Clézio. Une Analyse De La « Complexité Des Faits De Discours »
}

\author{
Okri Pascal Tossou \\ Maître de Conférences de Littérature française \\ Université d'Abomey- Calavi, Benin \\ Département des Lettres Modernes Laboratoire \\ de Recherches sur les Littératures Francophones
}

Doi: 10.19044/esj.2017.v13n29p158 URL:http://dx.doi.org/10.19044/esj.2017.v13n29p158

\begin{abstract}
J. M. G. Le Clézio represents one of the most eloquent contemporary French writers. This celebrity stems not only from a wide range of biography-based writings but mostly from the contents and aesthetics of his preferences. To me, Lullaby links up these two main facets which cristalize the singular character of Clézio productions. Firstly edited in 1978 by Gallimard in a book of short stories named Mondo et autres histoires, the text reveals itself as a youth novel by the same editor in 1980. Yet, this research apprehends it as a Bildungsroman, from its inner discourse, sharpened within the angle of initiatory convictions. To achieve such a goal, research tools include poetic narrative, sociocritics and discourse analysis.
\end{abstract}

Keywords : Lullaby, short story, Novel, generecity, initiation

\section{Résumé}

J. M. G. Le Clézio est l'un des plus grands écrivains français contemporain. Cette renommée s'est tissée non seulement autour du grand nombre de textes que sa biographie offre, mais surtout au regard de la pertinence de leur contenu et de ses choix esthétiques. Lullaby, à notre avis, charrie ces deux pans essentiels qui cristallisent la singularité de la création leclézienne. Paru d'bord en 1978 dans le recueil de nouvelles Mondo et autres histoires chez Gallimard, le texte se revendique roman de jeunesse chez le même éditeur en 1980. Néanmoins, notre étude l'envisage, à partir du discours qui l'irrigue, sous le prisme de convictions initiatiques qui en font un roman de formation. Pour ce faire, nous allions la Poétique narrative, la Sociocritique et l'Analyse du discours.

Mots-clés : Lullaby, nouvelle, roman, générécité, initiation 


\section{Introduction}

Plus que l'étiquette « genre » et les noms de genre qui ont tendance à réduire un énoncé à une catégorie de textes, la «généricité » se veut la mise en relation d'un texte avec des catégories génériques ouvertes. Jean-Michel Adam écrit à ce sujet:

La généricité permet de penser la participation d'un texte à plusieurs genres et cela est nécessaire car, à l'exclusion de genres socialement très contraints (déclaration d'impôts, procès verbal, rapport de thèse, etc.), de nombreux textes ne se conforment pas à un seul genre. Plutôt que de classer les textes dans une catégorie - leur appartenance -, il est intéressant d'observer les potentialités génériques qui les traversent - leur participation à un ou plusieurs genres. Analyser une participation au lieu de se limiter à une appartenance classificatoire permet d'entrer dans la complexité des faits de discours. ${ }^{1}$

C'est ce constat de la complexité des faits que nous faisons à la lecture de Lullaby ${ }^{2}$ de J.M.G. Le Clézio.

En effet, ce texte a d'abord paru comme une nouvelle dans le recueil Mondo et autres histoires avant d'être réédité sous forme de roman pour la jeunesse dans la collection Folio, ${ }^{3}$ Lullaby étant d'ailleurs un mot anglais signifiant berceuse, donc destiné à l'endormissement des enfants. Mais s'il est vrai qu'il existe des critères constants permettant de regrouper des textes sous un nom identique, des aspects d'un texte peuvent susciter d'autres niveaux de sens au lecteur. Pour Adam : «En parlant de généricité, il s'agit, par le suffixe -ité, de mettre en évidence le fait que les classes dont nous parlons ne sont que des potentialités attributives $\gg .{ }^{4}$ Mais, tout comme l'auteur de l'ouvrage qui déplace le statut du texte, peut-on tolérer le principe de généricité dans la réception de Lullaby afin de mieux penser le processus de lecture-interprétation ? S'il est vrai qu'un texte ne saurait prédéterminer toutes ses parentés ultérieures au moment de sa production, son identité générique classificatoire est-elle pour autant toujours ouverte? Nous nous intéressons au principe en vérifiant les motifs du glissement statutaire de

1 Jean-Michel Adam, «Discursivité, généricité et textualité, distinguer pour penser la complexité des faits de discours ", in Recherches $\mathrm{n}^{\circ} 56$, Les discours en classe de français, [en ligne], vu le 18/05/201, p. 12.

${ }^{2}$ J.M.G. LE CLEZIO, Lullaby, Paris, Gallimard, 1980.

${ }^{3}$ https://fr.wikipedia.org $>$ wiki $>$ Lullaby/09/05/17. Mondo et autres histoires est un recueil de nouvelles publié chez Gallimard en 1978. Anciennement figuré comme une nouvelle à l'intérieur d'un recueil, le texte paraît, seul, deux ans après, chez Gallimard, avec, en quatrième de couverture, l'indication suivante : «A partir de 11 ans. »

4 Jean-Michel Adam, «Discursivité, généricité et textualité, distinguer pour penser la complexité des faits de discours », in Recherches $\mathrm{n}^{\circ} 56$, Les discours en classe de français, p.11 [en ligne], consulté le 18/05/2017. 
Lullaby quand bien même l'ouvrage peut incarner, dans une certaine mesure, les différentes identités qu'il propose.

\section{Pourquoi de la nouvelle au roman de jeunesse ?}

«Depuis les années 1980, la critique universitaire ne cesse de s'enrichir, par de nombreuses thèses, par une session régulière annuelle au congrès du Conseil international d'études francophones (CIEF), par une revue, Les Cahiers J.M.G. Le Clézio (éditée par l'Association des lecteurs de Le Clézio créée en 2005), et par des colloques internationaux (tout d'abord en Espagne à Valence en 1992 et à Grenade en 2008, aux USA en 1997, au Maroc en 2004 puis en France à Toulouse en 2004 et à Arras en 2007, en comptant aussi une journée d'étude en 2002 dans les Yvelines). » ${ }^{5}$

C'est ainsi qu'Isabelle Roussel-Gillet ouvre le chapitre « Une œuvre plurielle, polyphonie des approches critiques ». Et pour cause. Quand à 23 ans Le Clézio recevait le Renaudot, c'était en partie grâce aux audaces esthétiques dans le roman, qui revendiquaient à la fois par les dispositions thématiques et typographiques les couleurs du Nouveau Roman. Les Géants, ${ }^{6}$ lourd roman où dessins, énoncés publicitaires, photographies, paragraphes barrés d'une croix et coupons de journaux cohabitent, reconduit le principe dix années plus tard. Et quand on se réfère à la forte interdiscursivité entre Désert et Poisson d'or, on ne peut pas ne pas s'interroger sur le glissement du statut générique de Lullaby opéré par l'écrivain qui déclasse cette nouvelle pour un roman de jeunesse. Car bien que Lullaby honore la caractéristique fondamentale de la nouvelle comme genre, notamment par le critère de la brièveté, ${ }^{7}$ et quand bien même a priori des traits établissant une identité générique à travers la nature référentielle du message, la longueur et le type de discours, etc. sont suffisants, on s'aperçoit que Lullaby est loin d'une «nouvelle amusante »8, en dépit, entre autres, de

${ }^{5}$ Isabelle Roussel-Gillet, J.M.G. Le Clézio, écrivain de l'incertitude, Paris, Ellipses Edition Marketing S.A, 2011, p. 89.

${ }^{6}$ Le Clézio, Les Géants, Paris, Gallimard, 1973.

7 « Seule la longueur, distinguerait donc la nouvelle du roman. D'autres critères peuvent être proposés. Mais se déduisent de l'étude des œuvres plutôt qu'ils ne s'imposent a priori. », Pierre-Louis Rey, Le Roman et la nouvelle, Collection Profil Histoire littéraire, Paris, Hatier, 2001, p. 87.

${ }^{8}$ Dans sa typologie des nouvelles, Godenne la définit comme celle à qui la « vie de tous les jours offre l'occasion de raconter des histoires divertissantes, amusantes, savoureuses, qui vont du bon mot, de la pochade sans grande portée, de la saynète de comédie de boulevard, de la scène de mœurs à la caricature parfois acerbe [...] Sujet piquant, situation cocasse, personnages drôles, humour tendre ou féroce, surprise finale (...) », René Godenne, $\mathrm{La}$ Nouvelle, Paris, Honoré Champion Editeur, 1995, p. 119. 
certaines dispositions typographiques qui lui confèrent par endroits une allure enfantine. Pour Nimier,

«Un roman est une aventure. Une aventure à la recherche d'un ordre [...] L'aventure finie, l'ordre se met de lui-même dans les choses. La dernière page, on s'en aperçoit tout à coup, était déjà dans la première. Il y a cette unité dans l'éparpillement, ce chant décidé à travers les rumeurs et cette sorte d'inflexible fidélité, la vie. $»^{9}$

Or, qu'il nous souvienne que Le Clézio est auteur de plus de cinquante ouvrages où romans, essais et nouvelles se répondent sur fond de cultures occidentale, mexicaine, amérindienne, maghrébine, mauricienne. Au contact de Lullaby, on découvre que le péritexte le prédispose comme un roman de jeunesse, notamment à travers la mention «A partir de 11 ans» portée au prière d'insérer du livre. Cette borne d'état civil, on s'en doute, verse fondamentalement le projet de l'ouvrage : il revendique un statut de roman de jeunesse, comme le certifie d'ailleurs le dessin de Lemoine en première de couverture. Déjà à la page 4, on pouvait lire que Georges Lemoine

« auteur de la couverture et des illustrations de Lullaby [...] est considéré comme l'un des plus grands dessinateurs de notre temps. Il a illustré de nombreux livres pour les enfants [...] Pour Folio Junior, Georges Lemoine est l'auteur des illustrations de La Maison qui s'envole de Claude Roy, de La Vie de Notre Seigneur Jésus-Christ de Charles Dickens et de L'Algérie ou la mort des autres de Virginie Buisson etc. $»^{10}$

En dehors de cette illustration de la première de couverture ${ }^{11}$, on peut évoquer celles des pages 33, 36, 39, 54 et 65 à l'intérieur de l'ouvrage. Par ailleurs, des indices internes au récit confortent cette classification. Il s'agit des personnages (Lullaby et le petit garçon pêcheur), les lexiques de début de lettres de Lullaby à son père, ${ }^{12}$ avec des dires de la classe de l'enfance comme «Peut-être que je fais un peu de bêtises » (p. 24), «Les filles sont bêtes à pleurer! Les garçons sont niais ! Ils n'aiment que leurs motos et leurs blousons !» (p. 25); des attitudes infantiles telles que : «Lullaby s'arrêta d'écrire. Elle mordilla un instant le bout de son Bic bleu, en regardant la feuille de papier à lettres $»^{13}$ ou, plus loin : «Elle enfourna dans

\footnotetext{
${ }^{9}$ Roger Nimier, Les Ecrivains sont-ils bêtes ?, Paris, Editions Payot \& Rivages, 2012, p. 19.

10 Soulignons que Lemoine propose beaucoup plus d'illustrations dans La Maison qui s'envole.

${ }^{11}$ Elle est répétée dans le texte à la page 15.

12 Première lettre : «Bonjour Ppa ». Puis une deuxième lettre toujours typographiquement «non alignée » qui s'étend cette fois de la page 24 à la page 27.

${ }^{13}$ Lullaby, op. cit., p. 11.
} 
son sac tout ce qu'elle trouva autour d'elle, sur la table et sur la chaise : rouge à lèvres, mouchoirs de papier, crayon à bille, clés, tube d'aspirine. ${ }^{14}$

Mais dans le même temps, des aspects de la diégèse se désolidarisent des fondements du roman de jeunesse qui, on le sait, féconde les dimensions esthétique et éthique du fait littéraire. Dans Lullaby, en effet, c'est la résolution de l'héroïne de rompre avec l'école, dès l'incipit, qui paraît curieuse. Ainsi, alors qu'il semble revendiquer par des aspects esthétiques un lectorat de jeunesse, le texte proclame par endroits une telle philosophie qu'on peut y soupçonner un autre projet esthétique aux frontières d'un trope communicationnel. ${ }^{15}$

\section{Du roman de jeunesse au bildungsroman ?}

A la lecture de Lullaby, l'horizon d'attente qui autorise à en espérer les déterminants d'une nouvelle se trouve perturbé. Et cela, tant par l'option formelle de l'ouvrage que par le fond, au point de lui conférer une hybridité générique et sémantique. Nous indiquerons certains aspects de ce jeu, que permettent de percevoir la dimension sémantique de l'effet-sujet et la fonction de régie du narrateur $;^{16}$ cela, par l'allégeance du discours à la nature et par des attributs du bildungsroman.

\section{D’abord, une allégeance à la nature}

Quand on se rappelle l'attachement de Le Clézio à l'environnement, ${ }^{17}$ on peut supposer que c'est ce liant qui tisse intégralement Lullaby,

${ }^{14}$ Idem., p.12.

15 "Il y a «trope communicationnel » chaque fois que s'opère, sous la pression du contexte, un renversement de la hiérarchie normale des destinataires ; c'est-à-dire chaque fois que le destinataire qui en vertu des indices d'allocution fait en principe figure de destinataire direct, ne constitue en fait qu'un destinataire secondaire, cependant que le véritable allocutaire, c'est en réalité celui qui a en apparence statut de destinataire indirect »", Catherine Kerbrat-Orecchioni, Les Interactions verbales, tome 1, Paris, Armand Colin, 1990, p. 92.

${ }^{16}$ Vincent Jouve, La Poétique du roman, Armand Colin/ VUEF, 2001. Le premier, «produit par un récit» (la subjectivité de son énonciateur) se laisse appréhender sur les plans sémantique (le contenu), syntaxique (l'organisation) et pragmatique ( la façon dont le destinataire est pris à parti) », p. 73 ; le second renvoie au discours métanarratif du narrateur pour marquer les articulations du récit, les connections, les inter-relations, bref l'organisation interne de la narration: voir Genette, Figures III, Paris, Editions du Seuil, 1972, pp. 261-262. Jouve précise qu'elle « consiste à organiser le récit. C'est elle qui permet les retours en arrière, les sauts en avant, les ellipses, les oppositions et les symétries. » p. 27. 17 «Depuis 1966, la France a fait exploser plus de 130 bombes nucléaires - certaines à l'air libre - dans les deux atolls de Mururoa et de Fangataufa. [...] La reprise des essais nucléaires par la France, à la fin de cet été, est à la fois un désastre écologique et une indignité morale. [...] Le Président saura-t-il entendre la voix des nations sans importance, écouter leur désespoir jusqu'à maintenant négligé ? », «Pour en finir avec le colonialisme nucléaire », Le Monde, $\mathrm{n}^{\circ} 15766, \mathrm{du}$ 04/10/1995, p. 15. Ou encore s'insurgeant contre le « raid » de Thierry 
notamment avec la grande inscription de la nature par les signifiants ciel, mer, soleil, horizon, colline, rochers, verts, vagues... qui coudent le texte. On se souvient que la découverte de la culture et du peuple amérindien dans les années $1970,{ }^{18}$ (les Emberas et leurs cousins germains, les Waunanas), dans la province du Darién au Panamá, réorienta sa création. Haï en reflète de larges pans. ${ }^{19}$ Dans La Fête chantée, livre de confidences de son contact avec ce peuple, le verbe «apprendre » est conjugué longtemps au creux de nombreux métissages: «(...) j’ai appris une nouvelle façon de voir, de sentir, de parler. [...] J'ai appris à reconnaître les plantes à leur odeur, leur saveur, les plantes à parfum $\gg .{ }^{20}$ Alors dans Lullaby, l'allégeance à la nature démarre dès le paragraphe incipitaire où «matin », « soleil », « morceau de ciel bleu », « vent », « mer d'un bleu sombre »,... fondent dans un paysage qui paraît fascinant pour le personnage : «Lullaby regarda tout cela, et elle se sentit soulagée d'avoir décidé de ne plus aller à l'école. » ${ }^{21} \mathrm{C}^{\mathrm{e}} \mathrm{st}$ également le cas dès l'entame de la première lettre que la jeune fille adresse à son père : "Il fait beau aujourd'hui. Le ciel est comme j'aime très très bleu. Je voudrais bien que tu sois là pour voir le ciel. La mer aussi est très très bleue. Bientôt ce sera l'hiver. ${ }^{22}$ Cette allégeance se répand dans l'évocation de ce qu'il convient d'appeler dans le roman le sujet de la mer qui gagne presque toutes les pages de l'ouvrage, souvent avec plusieurs occurrences sur une même page. ${ }^{23}$ Le thème de la mer, à notre sens, par cette figuration intempestive, participe d'un but illocutoire ${ }^{24}$ dans le texte, celui de

Sabine Organisation à travers la forêt guyanaise, sur les fleuves Maroni et Oyapock, projet qu'il qualifie de "monstrueux », Le Clézio interpelle, tout indigné : «Comment ose-t-on affirmer tranquillement que le passage de cinquante engins motorisés et surpuissants lancés au maximum de leur vitesse sur les eaux des fleuves ne causera pas de dégâts ? » in "Un projet monstrueux », Le Monde, $\mathrm{n}^{\circ} 13151$, du 12/05/ 1987, p. 2. On peut lire aussi avec intérêt « Sauver les baleines grises de Californie », in Le Monde ${ }^{\circ} 15614$ du 8 avril 1995, p. 15.

${ }^{18}$ Lullaby a paru justement en 1978 comme nouvelle.

${ }^{19}$ J. M.G. Le Clézio, Haï, Genève, 1971. Par ailleurs, dans L'Extase matérielle, il écrit : «Sans le savoir, sans lutter, puisque je le veux, j'ai commencé le long voyage de retour vers le gel et le silence, vers la matière multiple, calme et terrible; sans le comprendre, mais en étant sûr que je le fais, j'ai commencé le long voyage religieux qui ne se terminera sans doute jamais. », Paris, Gallimard, 1966, p. 315.

${ }^{20}$ J.M.G. Le Clézio, La Fête chantée et autres essais de thème amérindien, Paris, Gallimard, 1997, pp. 11-12.

${ }^{21}$ Lullaby, op. cit., p. 9.

${ }^{22}$ Idem., p. 10.

${ }^{23}$ Cinq fois à la page 16,4 fois aux pages $17,31,54,59,3$ fois aux pages $26,32,37,42,50$ et 69 .

${ }^{24}$ John R. Searle, Sens et expression, études de théorie des actes de langages, Paris, Les éditions de Minuit, 1982, p. 42 ; p $\square$ ur lui, le but illocutoire, une composante de la force illocutoire qui est la résultante de plusieurs éléments, est cette intention plus précise d'influencer l'auditeur. Rappelons que J. L. Austin s'étend sur les procédés longuement dans 
persuader de son importance, vu que la fascination qu'il exerce sur le personnage supplante l'école, pourtant institution prestigieuse, et par ailleurs symbole de convictions occidentales qui prépare à l'assimilation :

«La jeune fille s'arrêta dans les rochers pour écouter la mer. Elle connaissait bien son bruit, l'eau qui clapote et se déchire, puis se réunit en faisant exploser l'air, elle aimait bien cela, mais aujourd'hui, c'était comme si elle l'entendait pour la première fois. Il n'y avait rien d'autre que les rochers blancs, la mer, le vent, le soleil. Lullaby ne pensait même plus à l'école. La mer est comme cela : elle efface ces choses de la terre parce qu'elle est ce qu'il y a de plus important au monde. [...] Alors Lullaby était bien. $»^{25}$

On le voit, la plénitude habite le personnage au contact de la fascination que des éléments de la nature exercent sur elle. C'est ce qu'établit forcément ici en fin d'extrait l'adverbe «bien », qui déjà modalisait le verbe «connaître » (donc la vraie science) dans la deuxième phrase du passage, et cela, à l'imparfait de l'indicatif.

\section{Du bildungsroman...}

Avec les travaux de Suleiman, le terme de «bildungsroman » s'emploie " pour désigner un type d'histoire qui met l'accent sur la formation ou l'«éducation-de-soi »du héros [...] » ${ }^{26}$ Nous trouvons qu'il fonctionne dans Lullaby comme un modèle heuristique si nous entendons l'initiation comme le parcours de la révélation par l'épreuve en vue de la formation qui permet d'accéder à la connaissance. C'est un rite de passage qui symbolise la naissance d'un être nouveau, où souffrances diverses, tortures et mutilations peuvent se prêter à vocation formatrice. Pour Régine Robin,

" le héros évolue de l'ignorance du vrai et de la passivité vers une connaissance du vrai, de soi et vers l'action. [...] Sur son chemin, surmontant un certain nombre d'épreuves, le sujet doit rencontrer la Vérité (adhérer à une doctrine) et c'est cette connaissance du vrai qui le transforme et lui permet d'agir. La quête du héros est une quête de certitude, et le passage de l'ignorance à la vérité prend la forme d'un «scénario initiatique »",27.

Quand dire, c'est faire, Paris, Editions du Seuil, 1970.

${ }^{25}$ Lullaby, op.cit., pp. 16-17.

${ }^{26}$ Suzan Suleiman, Le Roman à thèse ou l'autorité fictive, Paris, Puf Ecriture 1983, p. 94.

${ }^{27}$ Régine Robin, Le Réalisme socialiste, une esthétique impossible, Paris, Payot, 1986, p. 276. C'est nous qui soulignons. 
Sans vouloir verser dans une phénoménologie de rituels initiatiques, il faut admettre la dimension symbolique ${ }^{28}$ du parcours de Lullaby. En effet, quand le personnage décide de rompre avec l'école, ici vue comme monde moderne (espace d'aliénation) pour aller à la Maison grecque, il faut mesurer tous les ancrages historiques qui tissent ce topos qui fonctionne comme le chronotope $^{29}$ matriciel qui le transmue. Mais avant qu'elle puisse s'y rende, la route de l'aventure se propose à Lullaby comme la cave à épreuves. Car l'aventure, dès le départ, l'engagea à l'opposé de l'avenue bruyante qu'elle emprunta en sortant de chez elle, allant « dans la direction opposée, vers les collines et les rochers ${ }^{30}$ comme semble l'indiquer bien plus loin le dessin de la page 23 aux frontières d'une errance picaresque, elle qui, une fois au bord des rochers, «ne pensait plus du tout aux rues, aux maisons, aux voitures, aux motocyclettes $»,{ }^{31}$ symboles des sociétés modernes de consommation. Voici le dessin :

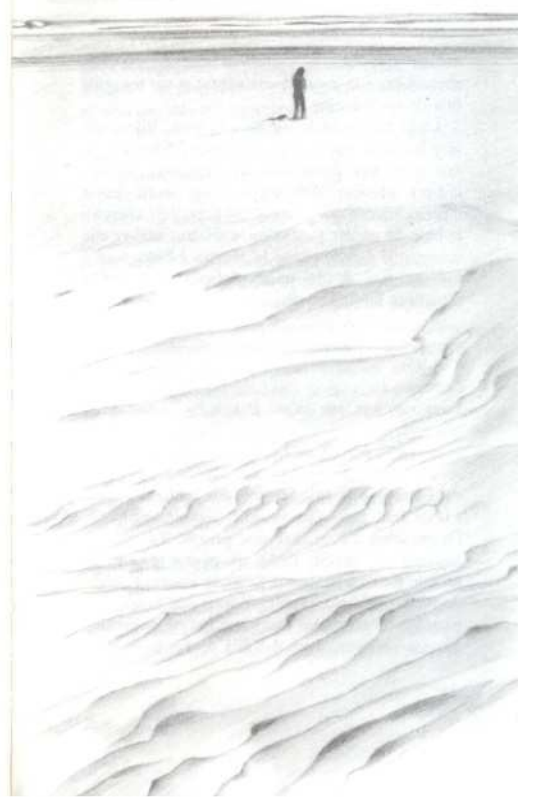

Mais l'expérience de la formation se complexifie sur le chemin des contrebandiers où il fallait "descendre le long d'un boyau étroit, sous la terre. Dans un tunnel, l'air froid fit frissonner la jeune fille. L'air était humide et sombre comme à l'intérieur d'une grotte. Les murs de la

\footnotetext{
${ }^{28}$ Un message est, entre autres, dit symbolique, écrit Pottier «si, par delà son sens manifeste, il est censé renvoyer à un sens latent auquel il est possible d'accéder au moyen d'une opération de décryptage. », in Richard Pottier, Anthropologie du mythe, Paris, Editions Kimé, 1994, p. 24.

${ }^{29}$ Mikhail Bakhtine, Esthétique et théorie du roman, Paris, Gallimard, 1978, pp. 237-238.

${ }^{30}$ Lullaby, op.cit., pp. 15-16.

${ }^{31}$ Idem., p. 17.
} 
forteresse sentaient le moisi et l'urine. ${ }^{32}{ }^{C}$ 'est seulement après que Lullaby tombe sur un énoncé énigmatico-initiatique sur le mur de la plate-forme, « en grandes lettres irrégulières $»$ :

«TROUVEZ-MOI » ${ }^{33}$

A l'analyse, on devine toute la charge initiatique de ce dire qui cumule les attributs de l'invitation et de l'impératif. C'est alors que Lullaby réalise que la quête de l'identité qui l'animait devenait de plus en plus « difficile ». Car sur cette route des Contrebandiers détruite, il "fallait escalader et sauter d'un rocher à l'autre, en s'aidant des mains pour ne pas glisser » ${ }^{34}$ sur cette route, il fallait surtout « calculer très vite du regard, voir les bons passages, les rochers qui font des escaliers ou des tremplins, deviner les chemins qui vous conduisent vers le haut; il faut éviter les culde-sac, les pierres friables, les crevasses, les buissons d'épines. ${ }^{35}$

Et c'est au creux de cette nécessité mathématique, qu'apparaît à l'héroïne son Professeur, comme c'est souvent le cas avec le néophyte et son Maître. Dans Lullaby, M. Fillipi est le Maître, totalement distinct dans la pensée de la jeune fille :

" Il était debout, en équilibre sur un rocher penché, et il souriait avec indulgence. Ses cheveux blancs faisaient une couronne dans la lumière du soleil, et derrière ses lunettes de myope, ses yeux bleus brillaient bizarrement. [...] «C'est très bien, très bien, mademoiselle », disait la voix de M. Fillipi dans son oreille. [ ...] Continuez comme cela, vous êtes sur la bonne voix. »"36

Ce sont donc les encouragements de M. Fillipi qui décideront Lullaby à mettre en application « les lois de Descartes » qui lui ont été jadis enseignées, et qui se proposent en fait comme une clé pour la résolution des équations du moment. Mais ce n'est pas tout : quand elle remonte à travers les rochers, la jeune néophyte voit pour la deuxième fois les signes énigmatiques produits à la craie sur les rochers, dont :

«NE VOUS DECOURAGEZ PAS !» «ÇA FINIT PEUT-ETRE EN QUEUE DE POISSON », ${ }^{37}$ avec, immédiatement dans le livre, un dessin à connotation frissonnante à la page 28 que voici :

\footnotetext{
${ }^{32}$ Idem., op. cit., p. 18.

${ }^{33}$ Idem., p. 19.

${ }^{34}$ Idem.

${ }^{35}$ Idem., pp. 19-20.

${ }^{36}$ Idem., p. 20-21.

${ }^{37}$ Idem., p. 27.
} 


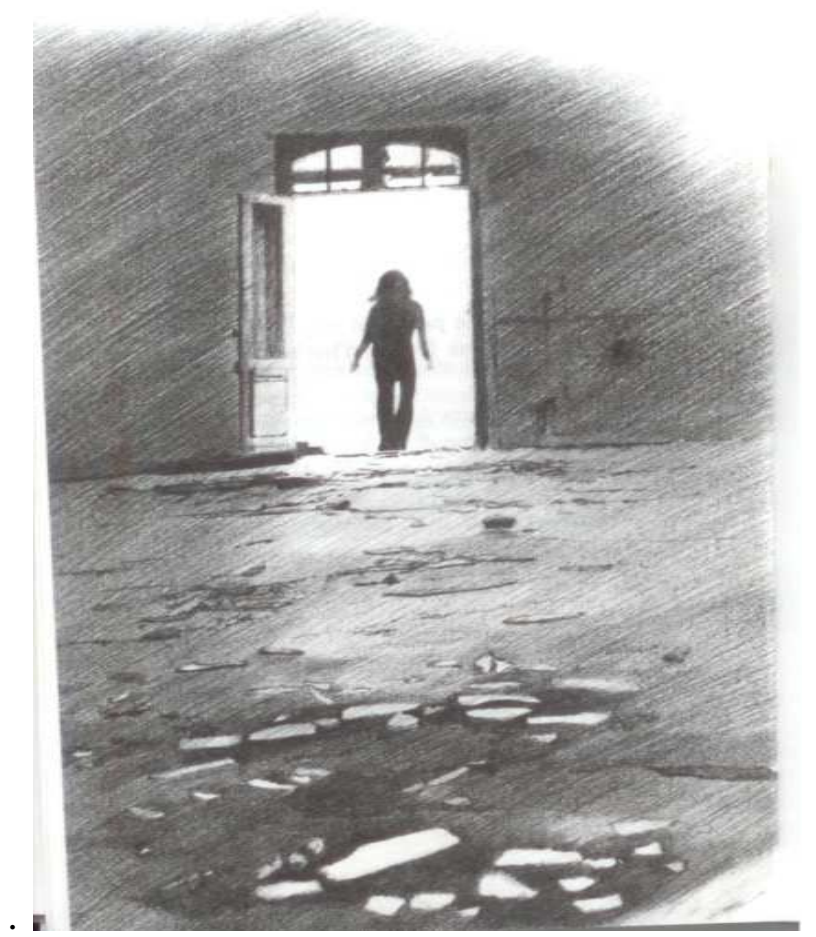

L'histoire montre par la suite au lecteur que

«Lullaby regarda à nouveau autour d'elle, mais il n'y avait personne dans les rochers, aussi loin qu'on puisse voir. Alors elle continua sa route. Elle grimpa, elle redescendit, elle sauta par-dessus les fissures, et à la fin elle arriva au bout du cap, là où il y avait un plateau de pierres, et la maison grecque. ${ }^{38}$

Cette Maison grecque s'offrait au personnage comme « un temple en miniature $»^{39}$ au beau nom fascinant, Karisma :

\section{XAPI $\Sigma$ MA}

L'intérieur du bâtiment était non seulement «sombre », mais aussi « froid». Et c'est dans cet univers d'indices manifestement étranges que, comme pour une identité retrouvée, Lullaby entend « une voix qui venait dans le vent », " voix très ancienne, qui avait traversé le ciel et la mer » et qui " " répétait son nom d'autrefois, le nom que son père lui avait donné un jour, avant qu'elle s'endorme. «Ariel...Ariel...»" ${ }^{40}$ C'est épisode du roman qui en établit significativement le projet esthétique, à savoir que la quête essentielle est identitaire. C'est pourquoi bien que Lullaby aille plus souvent

${ }^{38}$ Lullaby, op. cit., p. 29.

${ }^{39}$ Idem.

${ }^{40}$ Idem., p. 31. 
en ces lieux dans le chapitre 2, une sensation de questionnement la gagne à chaque coup :

«Quand Lullaby arrivait devant la maison, elle s'arrêtait, et son cour battait plus vite et plus fort, et elle sentait une chaleur étrange dans les veines de son corps, parce qu'il y avait sûrement un secret dans cet endroit. Le vent tombait d'un seul coup, et elle sentait toute la lumière du soleil qui l'enveloppait doucement, qui électrisait sa peau et ses cheveux. ${ }^{41}$

En ces lieux, le personnage vivra alors une espèce de métempsychose en plusieurs stations. En effet,

«la vie se retirait d'elle et partait, s'en allait dans le ciel et dans la mer. C'était difficile à comprendre, mais Lullaby était certaine que c'était comme cela, la mort. Son corps restait où il était, dans la position assise, le dos appuyé contre la colonne blanche, tout enveloppé de chaleur et de lumière. ${ }^{42}$

Car

«Il n'y avait presque plus de mouvements, presque plus de vie en elle, seulement son regard qui s'élargissait, qui se mêlait à l'espace comme un faisceau de lumière. Lullaby sentait son corps s'ouvrir, très doucement, comme une porte, et elle attendait de rejoindre la mer. ${ }^{43}$

Puis, par finir,

«Son corps ne devenait pas froid, comme sont les morts dans leurs chambres. La lumière continuait à entrer, jusqu'au fond des organes, jusqu'à l'intérieur des os, et elle vivait à la même température que l'air, comme les lézards. » ${ }^{44}$

Alors succède à cette «Renaissance», comme pour ravitailler son âme en nourritures spirituelles, la visite d'une autre maison grecque sur le site, où la surprend l'inconnu, « un homme vêtu d'un pantalon de toile bleue et d'un blouson, au visage noirci par le soleil, aux cheveux hirsutes. ${ }^{45}$ Dans sa fuite, Lullaby se tord la cheville, «boit le soleil », prit un bain de mer, s'échappe par la mer, ici symbole de purification par le motif d'eau salée. On comprend dès lors pourquoi M. Fillipi qualifie l'aventure de Lullaby de « voyage », et sa dernière parole excipitaire quand la jeune initiée, c'est le cas de le dire, le retrouve à l'école : « «Moi aussi j'aime la mer. »

Au total, après ces expériences de métempsychose, après donc l'eau (l'épisode de la baignade avec une eau «très transparente, froide » p. 22),

\footnotetext{
${ }^{41}$ Idem., p. 35.

${ }^{42}$ Idem., p. 37.

${ }^{43}$ Lullaby, op. cit., p. 38.

${ }^{44}$ Idem., p. 40.

${ }^{45}$ Idem., p. 52.
} 
l'air (vent de l'espace), la terre, (rocher, maison grecque), elle « avait envie de faire du feu». Pour que donc les quatre éléments cosmiques se recoupent :

"Elle avait envie de faire du feu. Elle chercha dans les rochers un endroit où le vent ne soufflerait pas trop fort. Un peu loin, elle trouva la petite crique avec l'embarcadère en ruine, et c'est là qu'elle s'installa. [...] A la base du rocher, il y avait un creux bien sec et chaud, et tout de suite les flammes s'élevèrent, légères, pâles, avec un froissement doux. ${ }^{46}$

\section{Conclusion}

La particularité d'un conte philosophique, on le sait, est qu'il féconde deux univers: derrière le récit apparent d'une histoire de mœurs, se proposent des enseignements philosophiques qui puisent à la fois dans l'ironie, l'hypotypose, l'éthopée, etc. pour offrir des tableaux d'exemplum ou d'inexemplarité. C'est le cas dans Zadig ou encore dans Candide $^{47}$ de Voltaire. Avec Lullaby, au-delà des attributs du genre de la nouvelle, c'est à ce type de texte que nous avons affaire. Seulement en plus de cette composante d'édification, cet ouvrage charrie les balises génériques traditionnelles au point d'en faire craquer les corsets: Lullaby était une nouvelle dans le recueil Mondo et autres histoires publié chez Gallimard en 1978 ; il se revendique roman de jeunesse toujours chez Gallimard en 1980. Et à l'issue de cette étude aujourd'hui, nous y percevons une charge initiatique loin de l'innocence infantile, mais fortement définitoire des spiritualités amérindiennes. Schaeffer a peut-être raison, qui écrit :

«Lorsqu'on étudie les relations entre textes et genres, on traite souvent les textes comme des objets physiques dotés d'une identité compacte. On en tire l'idée que la relation du texte au genre doit être la même dans tous les genres, ou, pour être plus précis, que toutes les déterminations génériques se réfèrent à des phénomènes textuels de même niveau et de même ordre. Or, lorsqu'on part des noms de genres, on est frappé au contraire par le fait que manifestement ils ne se réfèrent pas tous au même ordre de phénomènes. $~^{48}$

\footnotetext{
${ }^{46}$ Idem., p. 44.

${ }^{47}$ Candide aurait été réédité vingt fois du vivant de l'auteur : ce qui témoigne de son succès littéraire.

${ }^{48}$ Jean-Marie Schaeffer, Qu'est-ce qu'un genre littéraire?, Paris, Seuil, 1989, p. 79.
} 


\section{References:}

1. Jean-Michel ADAM, «Discursivité, généricité et textualité, distinguer pour penser la complexité des faits de discours », in Recherches $\mathrm{n}^{\circ} 56$, Les discours en classe de français. [en ligne], consulté le 18/05/2017.

2. J.L. AUSTIN, Quand dire, c'est faire, Paris, Editions du Seuil, 1970.

3. Mikhaïl BAKHTINE, Esthétique et théorie du roman, Paris, Gallimard, 1978.

4. Raphaël BARONI, "Généricité ou stéréotypie ? », Cahiers de Narratologie [En ligne], 17 | 2009, mis en ligne le 11 juillet 2011, consulté le 30 septembre 2016. URL : http://narratologie.revues.org/1090 ; $\quad$ DOI 10.4000/narratologie. 1090 .

5. Gérard GENETTE, Figures III, Paris, Seuil, 1972.

6. Vincent JOUVE, La Poétique du roman, Armand Colin/ VUEF, 2001.

7. Catherine KERBRAT-ORECCHIONI, Les Interactions verbales, tome 1, Paris, Armand Colin, 1990.

8. J.M.G. LE CLEZIO, -Le Procès-verbal, Paris, Gallimard, 1963.

9. L'extase matérielle, Paris, Gallimard, 1966.

10. Haï, Genève, Skira, 1971.

11. Les Géants, Paris, Gallimard, 1973.

12. Mondo et autres histoires, Paris, Gallimard, 1978.

13. Désert, Paris, Gallimard, 1980.

14. Lullaby, Paris, Gallimard, 1980.

15. «Un projet monstrueux », in Le Monde, $\mathrm{n}^{\circ} 13151$, du 12/05/ 1987

16. «Sauver les baleines grises de Californie », in Le Monde $\mathrm{n}^{\circ} 15614$, du 8 avril 1995, p. 15.

17. Poisson d'or, Paris, Gallimard, 1996.

18. La Fête chantée et autres essais de thème amérindien, Paris, Gallimard, 1997.

19. David MARTENS, Guillaume WILLEM, «Enjeux discursifs de la généricité des textes, Entretien avec Jean-Michel Adam », in Interférences littéraires/Literaire interferenties, $\mathrm{n}^{\circ}$ 13, juin 2014.

20. Roger NIMIER, Les Ecrivains sont-ils bêtes?, Paris, Editions Payot \& Rivages, 2012.

21. Richard POTTIER, Anthropologie du mythe, Paris, Editions Kimé, 1994.

22. Régine ROBIN, Le Réalisme socialiste, une esthétique impossible, Paris, Payot, 1986.

23. Isabelle ROUSSEL-GILLET, J.M.G. Le Clézio, écrivain de l'incertitude, Paris, Ellipses Edition Marketing S.A, 2011. 
24. Claude ROY, La Maison qui s'envole, Paris, Editions Gallimard Jeunesse, 1997.

25. Didier SAMAIN, «Linguistique ou Théorie du langage, généricité des concepts et axiomatisation des domaines », in Verbum XXXI, 2009, no 1-2, pp. 28-43.

26. Jean-Marie SCHAEFFER, Qu'est-ce qu'un genre littéraire?, Paris, Seuil, 1989.

27. John R. SEARLE, Sens et expression, études de théorie des actes de langages, Paris, Les éditions de Minuit, 1982.

28. Suzan SULEIMAN, Le Roman à thèse ou l'autorité fictive, Paris, Puf Ecriture 1983

29. VOLTAIRE, Zadig, Londres, Pour la compagnie, 1747.

30. Candide, Genève, Gabriel Cramer, 1759. 